\title{
Features of Hepatic Abscesses on Computed Tomography: Predicting the Outcomes of Percutaneous Catheter Drainage or Needle
} Aspiration

\author{
Soon Cheol Baek, ${ }^{1}$ Jisun Lee, ${ }^{1,}$ Min Ho Kang, ${ }^{1,}{ }^{*}$ Kyung Sik Yi, ${ }^{1}$ Yook Kim, ${ }^{1}$ Bum Sang Cho, ${ }^{1,2}$ Kil sun Park, ${ }^{1,2}$ \\ and Sang-Yong Eom ${ }^{3}$ \\ ${ }^{1}$ Department of Radiology, Chungbuk National University Hospital, Cheongju, South Korea \\ ${ }^{2}$ Department of Radiology, College of Medicine, Chungbuk National University, Cheongju, South Korea \\ ${ }^{3}$ Department of Preventive Medicine, College of Medicine, Chungbuk National University, Cheongju, South Korea \\ "Corresponding authors: Jisun Lee, Department of Radiology, Chungbuk National University Hospital, Cheongju, South Korea. Tel: +82-432696474, Fax: +8-432696479, E-mail: \\ dr.jisunlee@gmail.com; Min Ho Kang, Department of Radiology, Chungbuk National University Hospital, Cheongju, South Korea. Tel: +82-432696478, Fax: +82-432696479, \\ E-mail: sheepist@paran.com
}

Received 2016 July 09; Revised 2016 August 03; Accepted 2016 August 16.

\begin{abstract}
Background: Percutaneous catheter drainage (PCD) and percutaneous needle aspiration (PNA) have become the primary options for treating hepatic abscesses. Thus far, various studies have examined the clinical data and computed tomography (CT) features for predicting PCD outcomes.

Objectives: The goal was to compare CT features between hepatic abscesses classified based on strain and quantitative outcome of PCD or PNA, and to analyze clinical outcomes.

Patients and Methods: Eighty-seven patients who had undergone PCD or PNA with pyogenic hepatic abscess in a 5-year period were included. They were divided into Klebsiella pneumoniae monomicrobial pyogenic hepatic abscess (KHA) group and non- Klebsiella pneumoniae monomicrobial or polymicrobial pyogenic hepatic abscess (non-KHA) group. Patients were categorized into the following subgroups based on strain and percentage of initial drainage volume per CT volume: groups A (< 50\%) and B ( $\geq 50 \%)$, groups $\mathrm{C}$ and D in KHA group, and groups $\mathrm{E}$ and $\mathrm{F}$ in non-KHA group. We compared clinical findings, CT features, and clinical outcomes between the two groups, respectively.

Results: In univariate and multivariate analysis, patients with KHA group were associated with less frequent rim enhancement (P $=0.024)$. More frequent occurrence of thicker wall $(\mathrm{P}<0.001)$ and lower mean attenuation $(\mathrm{P}=0.047)$ were the most significant predictors in group $B$ and thicker wall $(\mathrm{P}=0.002)$ was the most significant predictor in group $\mathrm{D}$. Length of hospitalization in group D was significantly shorter than that in group $\mathrm{C}(\mathrm{P}=0.026)$.

Conclusion: Less frequent occurrence of rim enhancement on CT may serve as an indicator of KHA and presence of thick wall on CT may serve as a predictor of greater percentage of initial drainage amount per CT volume during PCD or PNA in hepatic abscess with a tendency of shorter length of hospital stay. Hence, CT findings may be useful for predicting the outcome of PCD or PNA in patients with hepatic abscess.
\end{abstract}

Keywords: Computed Tomography, Hepatic Abscess, Percutaneous Catheter Drainage, Klebsiella pneumoniae

\section{Background}

Among cases of pyogenic abscess, the most common causative organism is Escherichia coli; however, Klebsiella pneumoniae (K. pneumoniae) has recently been emerging as the most common causative organism in Asia $(1,2)$. Previous studies have shown that K. pneumoniae frequently affects patients with diabetes mellitus (DM) and often presents as metastatic infection $(2,3)$. Alsaif et al. (3) reported that hepatic abscesses involving K. pneumoniae appear as single, solid, or multiloculated hepatic abscesses, whereas Lee et al. (4) observed that thin wall and internal necrotic debris are useful CT features for the early diagno- sis of hepatic abscesses involving K. pneumoniae.

Percutaneous catheter drainage (PCD) and percutaneous needle aspiration (PNA) have become the primary options for treating hepatic abscesses due to the fewer complications and lower mortality associated, in comparison with those associated with surgery (5-9). Thus far, various studies have examined the clinical data and computed tomography(CT) features for predicting PCD outcomes (1012). However, no quantitative approach has been developed to assess the amount of pus drained through PCD. 


\section{Objectives}

In the present study, we aimed to compare CT features between hepatic abscesses classified specific groups based on the strain and quantitative outcome of PCD or PNA, and to analyze the clinical outcomes.

\section{Patients and Methods}

\subsection{Patients and Data Collection}

This retrospective study was approved by our institutional review board, and the need for written informed consent was waived. The study protocol conforms to the ethical guidelines of the 1975 declaration of Helsinki as reflected in a priori approval by the institutional human research committee. We performed a retrospective computer search of our hospital database records from March 2011 to March 2015 by using the international classification of diseases $\left(9^{\text {th }}\right.$ revision, code 572.0) to identify all patients at our institution who were diagnosed with pyogenic hepatic abscess; the database search yielded 218 patients. The inclusion criteria were presence of hepatic abscess in the liver on contrast-enhanced CT imaging prior to PCD or PNA, drainage of pus from the abscess cavity with PCD or PNA, and positive culture results from the hepatic abscess and/or blood cultures. Three patients who had not undergone contrast-enhanced CT imaging prior to PCD or PNA were excluded. Twenty-eight patients who underwent pus and blood culture examinations did not show positive findings and were excluded, whereas 100 patients who did not receive PCD or PNA during the hospitalization period were excluded. The population of the final study comprised 87 patients, including 55 men and 32 women, with a mean age of 63 years (range, 35 - 86 years).

Ultrasonographic (US) guidance used the Phillips iU 22 system with 5-MHz convex transducers for both PCD and PNA. PCD was performed regularly on abscesses with diameters greater than $3 \mathrm{~cm}$ unless the location of the abscess made it too difficult to drain. PNA was performed on abscesses with diameters less than $3 \mathrm{~cm}$ or inadequately liquefied abscesses on US. Patients underwent PCD in which a 10.2-Fr or 12-Fr pigtail catheter was placed into the largest abscess locule with the modified Seldinger technique by an expert radiologist with 10 years of experience. The size selection of drainage tubes was determined by the experienced radiologists at the time of drainage procedure. PNA was performed using a needle (16 Gauge). Once the needle tip or catheter was within the abscess cavity, it was aspirated until no more pus could be aspirated.

The hospital records were reviewed for demographic data; coexisting medical conditions, including DM, biliary disease, and malignancy; clinical symptoms, includ- ing fever and right upper quadrant (RUQ) abdominal pain; interval between symptom onset and final CT prior to the procedure, and the interval between symptom onset and the procedure day; initial laboratory data, including total white blood cell count, neutrophil count, and C-reactive protein level; amount of initial drainage; number of days the fever had subsided (body temperature of $\leq 37.5^{\circ} \mathrm{C}$ for 2 days), number of days leukocytosis had subsided (defined as a white blood count $<11000$ cells $/ \mathrm{mm}^{3}$ ), duration of drainage (defined as the interval between PCD tube insertion and removal), and length of hospitalization; and microbiological causes of hepatic abscess.

Patients were assigned to two groups according to the bacterial etiology: the K. pneumoniae monomicrobial pyogenic hepatic abscess (KHA) group and the non-K. pneumoniae monomicrobial or polymicrobial pyogenic hepatic abscess (non-KHA) group. A similar classification was used by Hui et al. (13). A monomicrobial hepatic abscess is an abscess wherein only one organism is recovered from the abscess aspirate and/or blood culture. A polymicrobial hepatic abscess is an abscess wherein more than one organism (one of which could be K. pneumoniae) is recovered from the abscess aspirate and/or blood culture.

In order to analyze the $\mathrm{CT}$ features and clinical outcomes according to the percentage of initial drainage volume per CT volume of hepatic abscess, we divided the patients into two groups: group A (percentage of initial drainage volume per CT volume $<50 \%, \mathrm{n}=41$ ) and group $\mathrm{B}$ (percentage of initial drainage volume per CT volume $\geq 50 \%, \mathrm{n}=46$ ). Moreover, the KHA group and non-KHA group were categorized into the following groups according to the percentage of initial drainage volume per CT volume: group $\mathrm{C}$ (percentage of initial drainage volume per CT volume $<50 \%$ ) and group D (percentage of initial drainage volume per CT volume $\geq 50 \%$ ) for patients in the KHA group, and group $\mathrm{E}$ (percentage of initial drainage volume per CT volume $<50 \%$ ) and group $\mathrm{F}$ (percentage of initial drainage volume per CT volume $\geq 50 \%$ ) for patients in the non-KHA group (Figure 1).

\subsection{CT Technique}

Forty-five patients underwent dynamic CT and the other 42 patients underwent single-phase CT. Unenhanced 64-slice multiple detector computed tomography (MDCT) scanner (Brilliance CT 64 Channel, Philips Medical, Eindhoven, The Netherlands) were routinely performed in these patients without any prior oral administration of water or contrast material. A total of $1.5 \mathrm{~mL} / \mathrm{kg}$ body weight of nonionic contrast material (Iomeprol, Bracco, Milan, Italy) was injected into an antecubital vein at a rate of $3.0 \mathrm{~mL} / \mathrm{s}$ by using a power injector. The scan delay for the arterial phase images was defined using bolus tracking, with a circular 


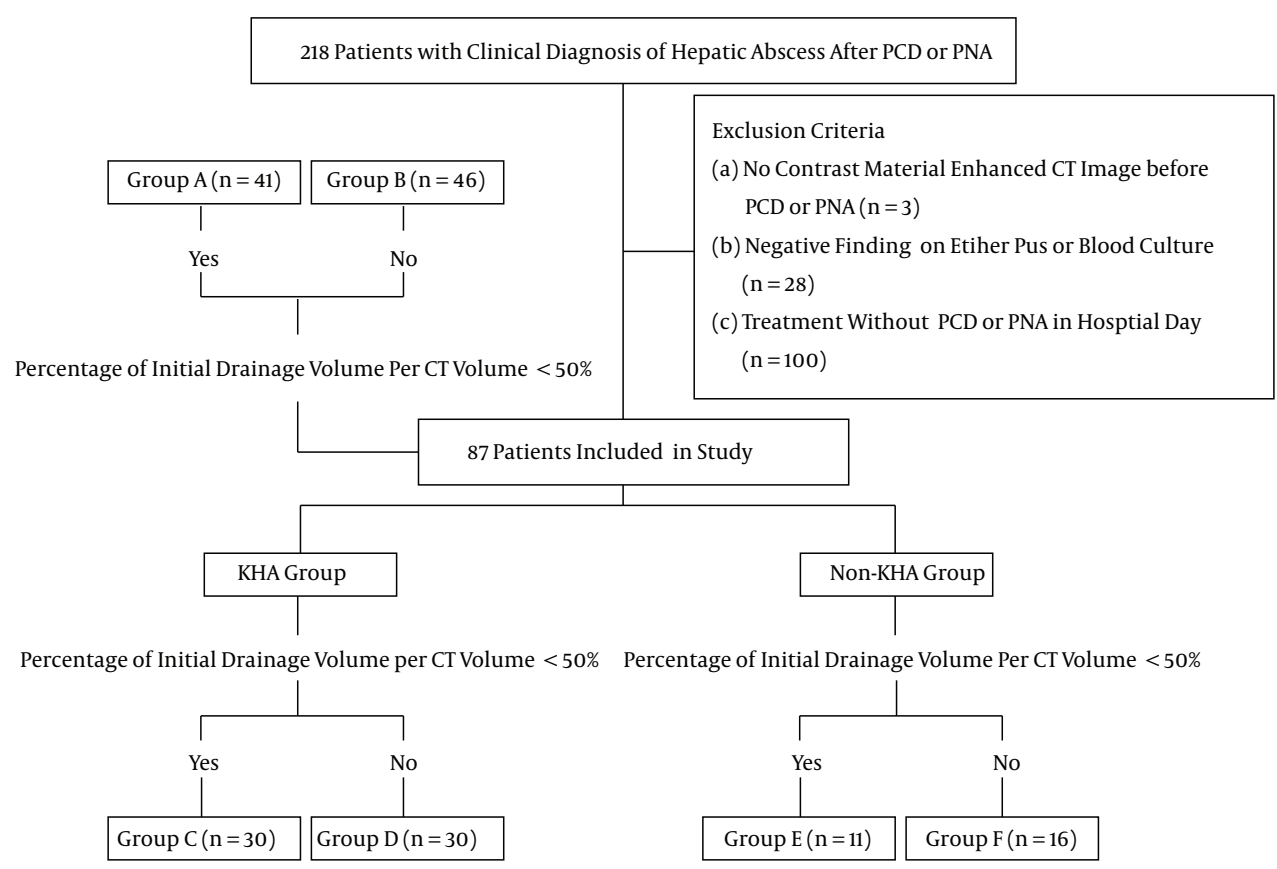

Figure 1. Study flow chart. According to the percentage of initial drainage volume per CT volume of hepatic abscess, we divided the patients into two groups: group A and B. Moreover, the KHA group and non-KHA group were categorized into the following groups according to the percentage of initial drainage volume per CT volume: group C and D for patients in the KHA group, and group E and F for patients in the non-KHA group. Abbreviations: KHA; Klebsiella pneumonia with monomicrobial hepatic abscess, Non-KHA; non-Klebsiella pneumoniae monomicrobial or polymicrobial pyogenic hepatic abscess.

region of interest (ROI) positioned at the level of the abdominal aorta; a predefined enhancement threshold level of 120 hounsfield unit (HU) was set to trigger data acquisition. The portal phase images were obtained $60 \mathrm{~s}$ after the scanning of the arterial phase was initiated. We used the following CT parameters: detector configuration, $64 \times$ $0.625 \mathrm{~mm}$; section thickness, $3.0 \mathrm{~mm}$; reconstruction interval, $2 \mathrm{~mm}$; tabulation speed, $46.8 \mathrm{~mm}$ per rotation; effective amperage setting, $150 \mathrm{~mA}$; rotation time, $0.75 \mathrm{~s}$; tube voltage, $120 \mathrm{kVp}$; and matrix, $512 \times 512$.

\subsection{CT Interpretation}

All patients underwent contrast-enhanced CT prior to drainage of the pyogenic hepatic abscess. Three experienced abdominal radiologists (S.C.B., J.S.L., and M.H.K., with 3, 7, and 12 years of experience in abdominal imaging, respectively) retrospectively reviewed the CT images until consensus was achieved. The following features were recorded: location (right liver, left liver, or both sides); size (in cases with multiple abscesses, the largest was measured); number (single or multiple); configuration (unilocular or multilocular); solid or cystic appearance (50\% or more of the abscess cavity appears hypodense or liquefied, with an attenuation value of $\leq 20 \mathrm{HU}$ ) in most of sections of the abdominal cavity; rim enhancement (more than half of the margin had a higher attenuation than the surrounding liver on contrast imaging); wall thickness (thin or thick); presence of gas density in the abscess; margin (ill-defined or well-defined; ill-defined was used to describe cases where more than half of the margin was finely speculated); and shortest length to the liver capsule (the shortest distance between the largest abscess and the liver capsule).

The CT volume of the hepatic abscess was measured by visual inspection of the portal venous phase images on contrast-enhanced CT. The outer margin of the hepatic abscess -excluding the wall- was drawn by hand, the area of this ROI was measured, and the area was then multiplied by the thickness of the slices to obtain the total volume. Thereafter, the CT volume was calculated as the sum of the areas in each section multiplied by the thickness; the percentage of initial drainage volume per CT volume was then calculated based on these values.

Accordingly, we used the method of Liao et al. to measure the mean attenuation (14). Attenuation value measurements were made using a commercially available workstation (Marosis m-view, Infinitt, Seoul, Korea) with the picture archiving communication system (PACS). In brief, the mean attenuation values were measured in the same manner as the CT volume. The ROI areas were multi- 
plied by the mean attenuation values in each section, and these values were summed to calculate the total attenuation values. The total attenuation values were then divided by the sum of the ROI areas to determine the final mean attenuation values of the abscess.

- Section 1: Mean = M1, Area = A1.

- Section 2: Mean $=$ M2, Area $=$ A2 .

- Section 3: Mean $=$ M3, Area $=$ A3.

CT Volume of the abscess $=(\mathrm{A} 1+\mathrm{A} 2+\mathrm{A} 3 \ldots) \times$ thickness. Final mean attenuation values of the abscess $=$

$(\mathrm{M} 1 \times \mathrm{A} 1+\mathrm{M} 2 \times \mathrm{A} 2+\mathrm{M} 3 \times \mathrm{A} 3 \ldots .) /.(\mathrm{A} 1+\mathrm{A} 2+\mathrm{A} 3 \ldots)$

\subsection{Statistical Analysis}

We compared the clinical findings and CT features between the KHA and non-KHA groups; groups A and B; groups $\mathrm{C}$ and $\mathrm{D}$ in the KHA group; and groups $\mathrm{E}$ and $\mathrm{F}$ in the non-KHA group. To investigate the differences in the clinical outcomes between the groups, we compared and analyzed the duration of drainage, number of days the fever had subsided, number of days leukocytosis had subsided, and the length of hospitalization between the groups. The $\chi^{2}$ analysis or Fisher exact test was used for the assessment of categorical variables and the Student t test was used for the assessment of continuous variables. Variables with a P value of less than 0.2 on univariate analysis were included the multivariate logistic regression analysis to identify the most reliable findings for predicting a welldrained hepatic abscess. The Spearman correlation coefficient was used to analyze the association of the percentage of initial drainage volume per CT volume with the CT volume and mean attenuation. In order to investigate the consistency between the three radiologists for the measurement of mean attenuation and CT volume on CT imaging, we estimated the intraclass correlation coefficients (ICC). An ICC of 0.61-0.80 was considered as indicative of substantial agreement and an ICC of higher than 0.80 was indicative of nearly perfect agreement (15).

All statistical analyses were conducted using SPSS 23 (IBM, Armonk, NY, USA). A P value of less than 0.05 was regarded as statistically significant.

\section{Results}

4.1. Comparison Between Monomicrobial K. Pneumoniae Hepatic Abscesses and Others

Of the 87 patients with pyogenic hepatic abscesses, 60 were assigned to the KHA group (positive findings on pus culture only in 37 , both pus and blood culture in 20 , and blood culture only in three) and 27 were assigned to the non-KHA group (positive findings on pus culture only in 13 , both pus and blood culture in 13, and blood culture only in one).

Univariate analysis showed significant differences between the two groups with underlying malignancy and underlying biliary disease (Table 1). Underlying malignancy and biliary disease were more common in the non-KHA group than in the KHA group $(P=0.001$ and $P=0.006$, respectively). On $\mathrm{CT}$, the KHA group commonly exhibited multilocular abscesses $(93.3 \%, \mathrm{P}=0.031)$, whereas the nonKHA group commonly exhibited rim enhancement (81.5\%, $\mathrm{P}=0.008)$ and presence of gas $(25.9 \%, \mathrm{P}=0.028)$. There were no differences between the two groups in terms of location, size, number, appearance, wall thickness, margin, shortest length to the liver capsule, mean attenuation, and CT volume.

Multivariate analysis indicated that less frequent underlying malignancy (odds ratio $[\mathrm{OR}]=0.09, \mathrm{P}=0.001$ ) and less frequent rim enhancement $(\mathrm{OR}=0.21, \mathrm{P}=0.019)$ were the most significant predicting factors of KHA.

\subsection{Percentage of Drainage Volume Per CT Colum of the Abscess (Group A Vs. Group B)}

We divided the patients into group A (percentage of initial drainage volume per CT volume $<50 \%, \mathrm{n}=41$ ) and group $B$ (percentage of initial drainage volume per CT volume $\geq 50 \%, \mathrm{n}=46$ ). Univariate analysis did not show any significant difference between groups A and B in terms of clinical data and initial laboratory data. On CT, group B more commonly showed frequent cystic appearance $(97.8 \%, \mathrm{P}=0.001)$, thick walls $(91.3 \%, \mathrm{P}<0.001)$, rim enhancement $(73.9 \%, \mathrm{P}=0.009)$, and lower mean attenuation $(33.7 \pm 10.7, \mathrm{P}<0.001$; Table 2$)$. There were no differences between the two groups in terms of location, size, number, configuration, margin, shortest length to the liver capsule, and CT volume. Multivariate analysis indicated that the more frequent occurrence of a thicker wall $(\mathrm{OR}=18.39, \mathrm{P}<0.001)$ and lower mean attenuation $(\mathrm{OR}=$ $0.95, \mathrm{P}=0.047$ ) were the most significant predicting factors in group B (Figures 2 and 3). The percentage of initial drainage volume per CT volume was negatively correlated with mean attenuation (Spearman coefficient $=-0.41, \mathrm{P}<$ 0.001).

\subsection{Comparison Between the KHA Group with Poor Drainage and Good Drainage (Group C Vs. Group D)}

We divided the patients into group $\mathrm{C}$ (percentage of initial drainage volume per CT volume $<50 \%, \mathrm{n}=30$ ) and group $\mathrm{D}$ (percentage of initial drainage volume per CT volume $\geq 50 \%, n=30$ ) in the KHA group. Univariate analysis indicated that the KHA group with good drainage (group D) more commonly exhibited cystic appearance (96.7\%, P = 
Table 1. Clinical Findings, CT Features, and PCD Outcomes in KHA and Non-KHA Groups ${ }^{\mathrm{a}}$

\begin{tabular}{|c|c|c|c|}
\hline Characteristics & KHA Group $(n=60)$ & Non-KHA Group $(n=27)$ & P Value \\
\hline $\operatorname{Age}(y)^{b}$ & $62.3 \pm 13.5$ & $64.5 \pm 11.5$ & 0.462 \\
\hline Sex, male & $38(63.3)$ & $17(63.0)$ & 0.974 \\
\hline \multicolumn{4}{|l|}{ Symptom } \\
\hline Fever & $42(70.0)$ & $19(70.4)$ & 0.972 \\
\hline RUQ pain & $19(31.7)$ & $7(25.9)$ & 0.588 \\
\hline \multicolumn{4}{|l|}{ Underlying disease } \\
\hline Diabetes mellitus & $15(25.0)$ & $5(18.5)$ & 0.506 \\
\hline Malignancy & $4(6.7)$ & $13(48.2)$ & 0.001 \\
\hline Biliary disease & $13(21.7)$ & $16(59.3)$ & 0.006 \\
\hline Interval between symptom onset and final CT prior to the procedure (days) ${ }^{b}$ & $7.7 \pm 6.1$ & $7.2 \pm 7.1$ & 0.775 \\
\hline Interval between symptom onset and the procedure day (days) ${ }^{b}$ & $9.0 \pm 6.0$ & $8.5 \pm 7.2$ & 0.754 \\
\hline White blood cell count $\left(\mathbf{x 1 0}^{9} / \mathrm{L}\right)^{\mathrm{b}}$ & $14.5 \pm 7.8$ & $14.7 \pm 7.6$ & 0.927 \\
\hline Neutrophil count $\left(\mathbf{x 1 0}^{9} / \mathrm{L}\right)^{\mathrm{b}}$ & $12.7 \pm 7.7$ & $12.5 \pm 7.4$ & 0.926 \\
\hline C-reactive protein level $(\mathrm{mg} / \mathrm{dL})^{\mathrm{b}}$ & $26.5 \pm 34.3$ & $19.9 \pm 9.5$ & 0.173 \\
\hline $\operatorname{Size}(\mathbf{c m})^{b}$ & $6.9 \pm 2.2$ & $6.4 \pm 2.3$ & 0.371 \\
\hline Number & & & 0.124 \\
\hline Single & $49(81.7)$ & $18(66.7)$ & \\
\hline Multiple & $11(18.3)$ & $9(33.3)$ & \\
\hline Location & & & 0.211 \\
\hline Right & $41(68.3)$ & $19(70.4)$ & \\
\hline Left & $5(8.3)$ & $5(18.5)$ & \\
\hline Both & $14(23.3)$ & $3(11.1)$ & \\
\hline Configuration & & & 0.031 \\
\hline Unilocular & $4(6.7)$ & $7(25.9)$ & \\
\hline Multilocular & $56(93.3)$ & $20(74.1)$ & \\
\hline Appearance & & & 0.327 \\
\hline Cystic & $50(83.3)$ & $25(92.6)$ & \\
\hline Solid & $10(16.7)$ & $2(7.4)$ & \\
\hline Wall thickness & & & 0.878 \\
\hline Thin wall & $21(35.0)$ & $9(33.3)$ & \\
\hline Thick wall & $39(65.0)$ & $18(66.7)$ & \\
\hline Margin & & & 0.456 \\
\hline Ill-defined & $13(21.7)$ & $4(14.8)$ & \\
\hline Well-defined & $47(78.3)$ & $23(85.2)$ & \\
\hline Rim enhancement & $31(51.7)$ & $22(81.5)$ & 0.008 \\
\hline Presence of gas density & $5(8.3)$ & $7(25.9)$ & 0.028 \\
\hline The shortest length to the liver capsule $(\mathrm{cm})^{\mathrm{b}}$ & $0.5 \pm 1.1$ & $0.3 \pm 0.5$ & 0.206 \\
\hline Mean attenuation $(\mathrm{HU})^{\mathrm{b}}$ & $39.2 \pm 11.9$ & $38.2 \pm 17.5$ & 0.796 \\
\hline CT volume $(\mathbf{m L})^{\text {b }}$ & $103.6 \pm 211.3$ & $67.2 \pm 53.7$ & 0.216 \\
\hline Amount of initial drainage $(\mathbf{m L})^{b}$ & $36.5 \pm 36.8$ & $36.0 \pm 34.7$ & 0.956 \\
\hline Percentage of initial drainage volume per CT Volume (\%) & $51.2 \pm 35.7$ & $62.7 \pm 46.3$ & 0.209 \\
\hline
\end{tabular}

0.006), thick walls (93.3\%, P $<0.001)$, and lower mean attenuation $(34.2 \pm 10.0, \mathrm{P}=0.001)$ as compared to the KHA group with poor drainage (group C) (Table 3). Multivariate analysis indicated that the more frequent occurrence of a 
Table 2. Clinical Findings, CT Feature and PCD Outcomes in Groups A and $\mathrm{B}^{\mathrm{a}, \mathrm{b}, \mathrm{c}}$

\begin{tabular}{|c|c|c|c|}
\hline Characteristics & KHA Group $(n=60)$ & Non-KHA Group $(n=27)$ & P Value \\
\hline $\operatorname{Age}(y)^{d}$ & $62.3 \pm 13.5$ & $64.5 \pm 11.5$ & 0.462 \\
\hline Sex, male & $38(63.3)$ & $17(63.0)$ & 0.974 \\
\hline \multicolumn{4}{|l|}{ Symptom } \\
\hline Fever & $42(70.0)$ & $19(70.4)$ & 0.972 \\
\hline RUQ pain & $19(31.7)$ & $7(25.9)$ & 0.588 \\
\hline \multicolumn{4}{|l|}{ Underlying disease } \\
\hline Diabetes mellitus & $15(25.0)$ & $5(18.5)$ & 0.506 \\
\hline Malignancy & $4(6.7)$ & $13(48.2)$ & 0.001 \\
\hline Biliary disease & $13(21.7)$ & $16(59.3)$ & 0.006 \\
\hline Interval between symptom onset and final CT prior to the procedure (days) ${ }^{d}$ & $7.7 \pm 6.1$ & $7.2 \pm 7.1$ & 0.775 \\
\hline Interval between symptom onset and the procedure day (days) ${ }^{d}$ & $9.0 \pm 6.0$ & $8.5 \pm 7.2$ & 0.754 \\
\hline White blood cell count $\left(\mathrm{x10}^{9} / \mathrm{L}\right)^{\mathrm{d}}$ & $14.5 \pm 7.8$ & $14.7 \pm 7.6$ & 0.927 \\
\hline Neutrophil count $\left(\mathrm{x10}^{9} / \mathrm{L}\right)^{\mathrm{d}}$ & $12.7 \pm 7.7$ & $12.5 \pm 7.4$ & 0.926 \\
\hline C-reactive protein level $(\mathrm{mg} / \mathrm{dL})^{\mathrm{d}}$ & $26.5 \pm 34.3$ & $19.9 \pm 9.5$ & 0.173 \\
\hline $\operatorname{Size}(\mathbf{c m})^{\mathbf{d}}$ & $6.9 \pm 2.2$ & $6.4 \pm 2.3$ & 0.371 \\
\hline Number & & & 0.124 \\
\hline Single & $49(81.7)$ & $18(66.7)$ & \\
\hline Multiple & $11(18.3)$ & $9(33.3)$ & \\
\hline Location & & & 0.211 \\
\hline Right & $41(68.3)$ & $19(70.4)$ & \\
\hline Left & $5(8.3)$ & $5(18.5)$ & \\
\hline Both & $14(23.3)$ & $3(11.1)$ & \\
\hline Configuration & & & 0.031 \\
\hline Unilocular & $4(6.7)$ & $7(25.9)$ & \\
\hline Multilocular & $56(93.3)$ & $20(74.1)$ & \\
\hline Appearance & & & 0.327 \\
\hline Cystic & $50(83.3)$ & $25(92.6)$ & \\
\hline Solid & $10(16.7)$ & $2(7.4)$ & \\
\hline Wall thickness & & & 0.878 \\
\hline Thin wall & $21(35.0)$ & $9(33.3)$ & \\
\hline Thick wall & $39(65.0)$ & $18(66.7)$ & \\
\hline Margin & & & 0.456 \\
\hline Ill-defined & $13(21.7)$ & $4(14.8)$ & \\
\hline Well-defined & $47(78.3)$ & $23(85.2)$ & \\
\hline Rim enhancement & $31(51.7)$ & $22(81.5)$ & 0.008 \\
\hline Presence of gas density & $5(8.3)$ & $7(25.9)$ & 0.028 \\
\hline The shortest length to the liver capsule $(\mathrm{cm})^{d}$ & $0.5 \pm 1.1$ & $0.3 \pm 0.5$ & 0.206 \\
\hline Mean attenuation $(\mathrm{HU})^{\mathrm{d}}$ & $39.2 \pm 11.9$ & $38.2 \pm 17.5$ & 0.796 \\
\hline CT volume $(\mathrm{mL})^{\mathbf{d}}$ & $103.6 \pm 211.3$ & $67.2 \pm 53.7$ & 0.216 \\
\hline Amount of initial drainage $(\mathrm{mL})^{\mathrm{d}}$ & $36.5 \pm 36.8$ & $36.0 \pm 34.7$ & 0.956 \\
\hline Percentage of initial drainage volume per CT Volume $(\%)^{d}$ & $51.2 \pm 35.7$ & $62.7 \pm 46.3$ & 0.209 \\
\hline $\begin{array}{l}\text { Abbreviations: RUQ; right upper quadrant, HU; hounsfield unit; PCD, Percutaneous c } \\
\text { a Values are expressed as No. (\%) unless otherwise indicated, percentages are calculat } \\
\text { b Percentage of initial drainage volume per CT volume }<50 \% \\
{ }^{b} \text { Percentage of initial drainage volume per CT volume } \geq 50 \% \\
{ }^{d} \text { Data are means } \pm \text { standard deviations }\end{array}$ & $\begin{array}{l}\text { ge; y, year. } \\
\text { of each group. }\end{array}$ & & \\
\hline
\end{tabular}

thicker wall $(\mathrm{OR}=15.11, \mathrm{P}=0.002)$ was the most significant predicting factor in the KHA group with good drainage 


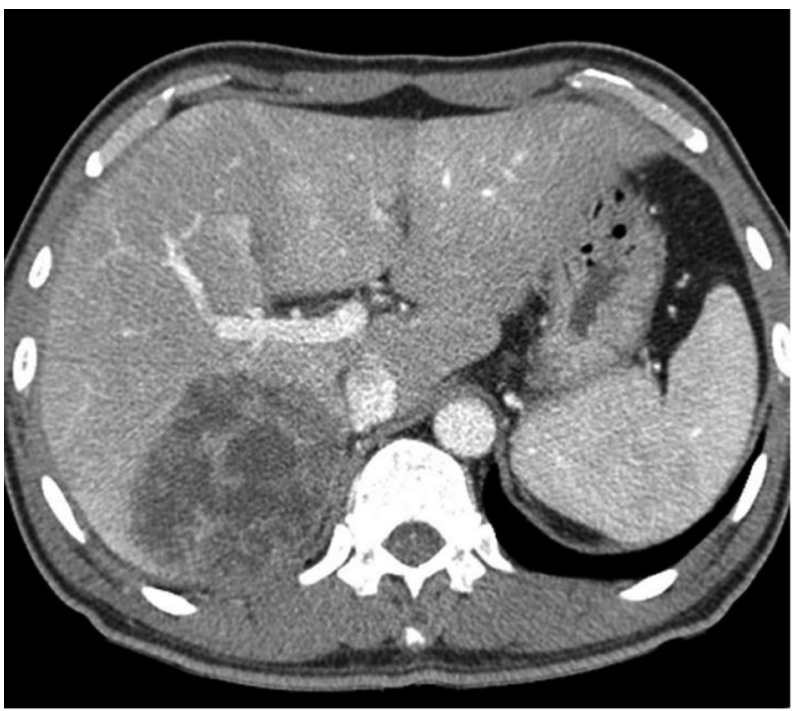

Figure 2. A 50-year-old man with Klebsiella pneumoniae hepatic abscess in group A. Contrast enhanced CT scan shows a single hepatic abscess in the right liver. It is a well-defined, multiloculated, and thin-walled abscess with no rim enhancement. The abscess has a multiple septa and solid appearance with less than $50 \%$ liquefied part of the abscess. The mean attenuation was calculated as 47 hounsfield unit (HU).

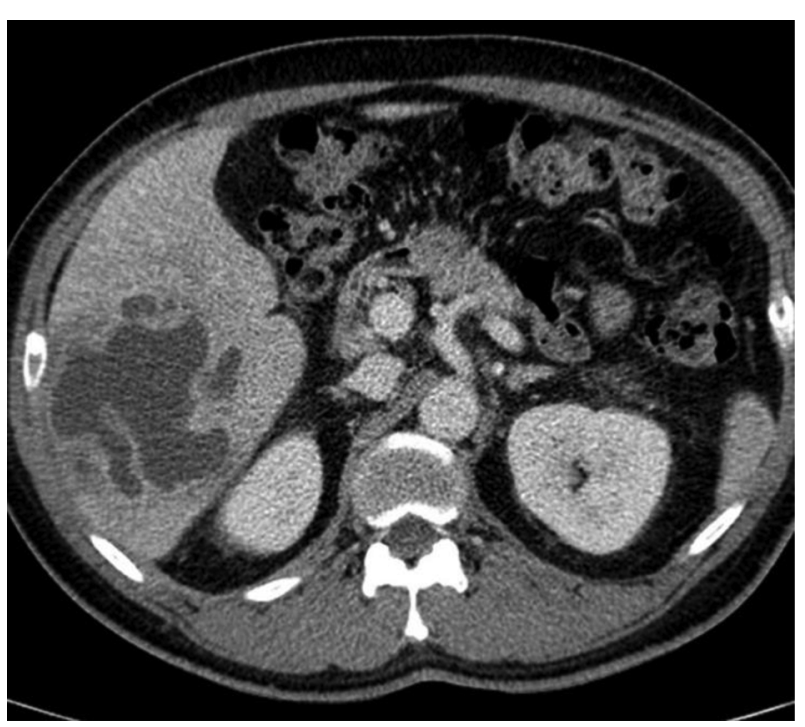

Figure 3. A 46-year-old man with Klebsiella pneumoniae hepatic abscess in group B Contrast enhanced CT scan shows a single multiloculated abscess with cystic appearance, thick wall, and rim enhancement in the right liver. The mean attenuation was calculated as 10 hounsfield unit (HU)

(group D, Figures 4 and 5).

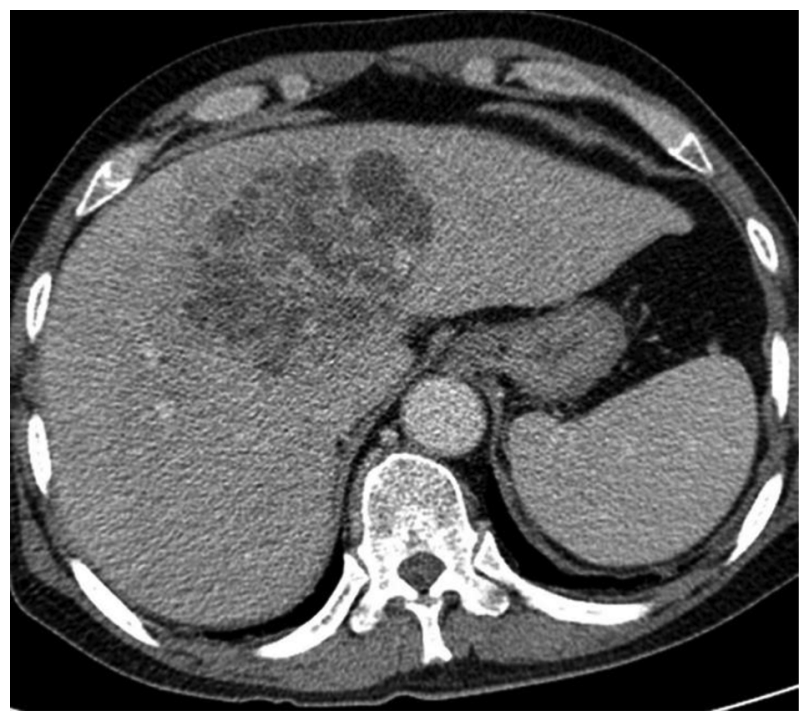

Figure 4. An 81-year-old man with Klebsiella pneumoniae hepatic abscess in group C. Contrast enhanced CT scan shows a single hepatic abscess with no rim enhancement. The abscess has a solid appearance and thin rim. The mean attenuation was calculated as 47 hounsfield unit (HU).

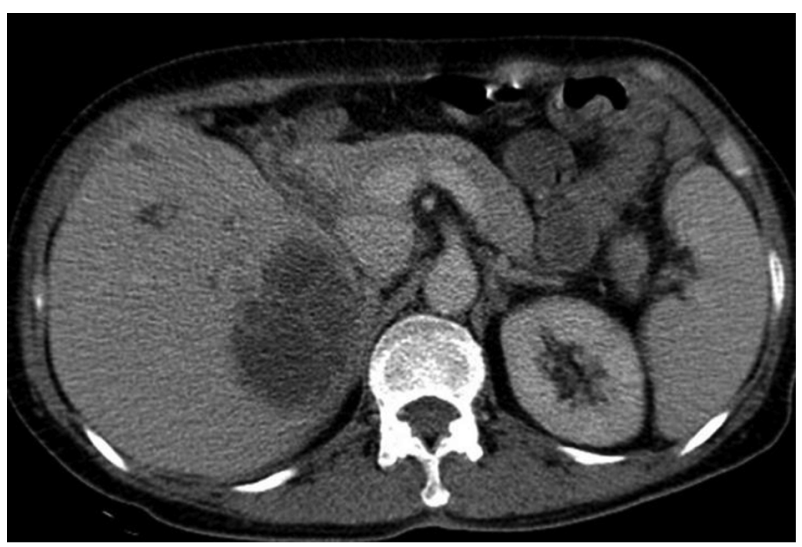

Figure 5. A 61-year-old man with Klebsiella pneumoniae hepatic abscess in group D. Contrast enhanced CT scan shows a single multiloculated abscess with cystic appearance, and thick wall in the right liver. The mean attenuation was calculated as 20 hounsfield unit (HU). The abscess was successfully treated with percutaneous catheter drainage. The patient was discharged from hospital 29 days later.

4.4. Comparison Between the Non-KHA Group with Poor Drainage and Good Drainage (Group E Vs. Group F)

We divided the patients into group $\mathrm{E}$ (percentage of initial drainage volume per CT volume $<50 \%, \mathrm{n}=11$ ) and group $\mathrm{F}$ (percentage of initial drainage volume per CT volume $\geq 50 \%, n=16$ ) in the non-KHA group. Univariate analysis indicated that the non-KHA group with good drainage (group F) more commonly showed a thicker wall (87.5\%, P $=0.012)$ and rim enhancement $(100.0 \%, \mathrm{P}=0.006)$ as com- 
Table 3. CT Features and PCD Outcomes in Groups $C$ and $D^{\mathrm{a}}$

\begin{tabular}{|c|c|c|c|}
\hline Characteristics & Group C $^{\mathbf{b}}(\mathbf{n}=\mathbf{3 0})$ & Group $D^{c}(n=30)$ & P Value \\
\hline $\operatorname{Size}(\mathbf{c m})^{d}$ & $7.0 \pm 2.4$ & $6.8 \pm 2.0$ & 0.808 \\
\hline Number & & & 0.739 \\
\hline Single & $24(80.0)$ & $25(83.3)$ & \\
\hline Multiple & $6(20.0)$ & $5(16.7)$ & \\
\hline Location & & & 0.894 \\
\hline Right & $21(70.0)$ & $20(66.7)$ & \\
\hline Left & $2(6.7)$ & $3(10.0)$ & \\
\hline Both & $7(23.3)$ & $7(23.3)$ & \\
\hline Configuration & & & 0.612 \\
\hline Unilocular & $3(10.0)$ & $1(3.3)$ & \\
\hline Multilocular & $27(90.0)$ & $29(96.7)$ & \\
\hline Appearance & & & 0.006 \\
\hline Cystic & $21(70.0)$ & $29(96.7)$ & \\
\hline Solid & $9(30.0)$ & $1(3.3)$ & \\
\hline Wall thickness & & & $<0.001$ \\
\hline Thin wall & $19(63.3)$ & $2(6.7)$ & \\
\hline Thick wall & $11(36.7)$ & $28(93.3)$ & \\
\hline Margin & & & 1.000 \\
\hline Ill-defined & $7(23.3)$ & $6(20.0)$ & \\
\hline Well-defined & $23(76.7)$ & $24(80.0)$ & \\
\hline Rim enhancement & $13(43.3)$ & $18(60.0)$ & 0.197 \\
\hline Presence of gas density & $3(10.0)$ & $2(6.7)$ & 1.000 \\
\hline The shortest length to the liver capsule $(\mathrm{cm})^{d}$ & $0.7 \pm 1.4$ & $0.3 \pm 0.6$ & 0.194 \\
\hline Mean attenuation $(\mathrm{HU})^{\mathrm{d}}$ & $44.1 \pm 11.8$ & $34.2 \pm 10.0$ & 0.001 \\
\hline CT volume $(\mathrm{mL})^{\mathrm{d}}$ & $138.7 \pm 291.8$ & $68.4 \pm 55.5$ & 0.204 \\
\hline
\end{tabular}

Abbreviations: HU; hounsfield unit; PCD, Percutaneous catheter drainage.

${ }^{a}$ Values are expressed as No. (\%) unless otherwise indicated, percentages are calculated on the basis of each group.

${ }^{b}$ Percentage of initial drainage volume per CT volume $<50 \%$

${ }^{c}$ Percentage of initial drainage volume per CT volume $\geq 50 \%$

${ }^{\mathrm{d}}$ Data are means \pm standard deviations.

pared to the non-KHA group with poor drainage (group E) (Table 4). However, multivariate analysis did not show any significant differences in the CT findings between the 2 groups.

\subsection{Clinical Outcome Among the Groups}

The length of hospitalization in the KHA group with good drainage (group D) was significantly shorter than that in the KHA group with poor drainage (group C; $\mathrm{P}=$ 0.026; Table 5). Although the length of hospitalization in the group with good drainage (group B) was shorter than that in the group with poor drainage (group A), the value was not significantly different. Moreover, the other clinical outcomes did not significantly differ between the groups.

The inter-observer variability for the measurement of mean attenuation and CT volume was excellent, with an intra-class correlation coefficient of 0.99 and 0.99 , respectively.

\section{Discussion}

We found that the rim enhancement of abscesses in the KHA group was not a significant finding as compared to that in non-KHA group. According to previous studies, pyogenic abscesses typically show peripheral rim en- 
Table 4. CT Features and PCD Outcomes in Groups $\mathrm{E}$ and $\mathrm{F}^{\mathrm{a}}$

\begin{tabular}{|c|c|c|c|}
\hline Characteristics & Group E $E^{b}(n=11)$ & Group $F^{c}(n=16)$ & P Value \\
\hline $\operatorname{Size}(\mathrm{cm})^{\mathrm{d}}$ & $6.7 \pm 2.2$ & $6.3 \pm 2.3$ & 0.668 \\
\hline Number & & & 1.000 \\
\hline Single & $7(63.6)$ & $11(68.8)$ & \\
\hline Multiple & $4(36.4)$ & $5(31.3)$ & \\
\hline Location & & & 0.300 \\
\hline Right & $9(81.8)$ & $10(62.5)$ & \\
\hline Left & $2(18.2)$ & $3(18.8)$ & \\
\hline Both & 0 & $3(18.8)$ & \\
\hline Configuration & & & 0.391 \\
\hline Unilocular & $4(36.4)$ & $3(18.8)$ & \\
\hline Multilocular & $7(63.6)$ & $13(81.3)$ & \\
\hline Appearance & & & 0.157 \\
\hline Cystic & $9(81.8)$ & $16(100.0)$ & \\
\hline Solid & $2(18.2)$ & 0 & \\
\hline Wall thickness & & & 0.012 \\
\hline Thin wall & $7(63.6)$ & $2(12.5)$ & \\
\hline Thick wall & $4(36.4)$ & $14(87.5)$ & \\
\hline Margin & & & 0.273 \\
\hline Ill-defined & $3(27.3)$ & $1(6.3)$ & \\
\hline Well-defined & $8(72.7)$ & $15(93.8)$ & \\
\hline Rim enhancement & $6(54.6)$ & $16(100.0)$ & 0.006 \\
\hline Presence of gas density & $2(18.2)$ & $5(31.3)$ & 0.662 \\
\hline The shortest length to the liver capsule $(\mathrm{cm})^{\mathrm{d}}$ & $0.4 \pm 0.6$ & $0.2 \pm 0.5$ & 0.518 \\
\hline Mean attenuation $(\mathrm{HU})^{\mathrm{d}}$ & $46.3 \pm 21.5$ & $32.6 \pm 12.0$ & 0.075 \\
\hline CT volume $(\mathrm{mL})^{\mathrm{d}}$ & $70.6 \pm 61.8$ & $64.8 \pm 49.4$ & 0.787 \\
\hline
\end{tabular}

Abbreviations: HU; hounsfield unit; PCD, Percutaneous catheter drainage.

${ }^{a}$ Values are expressed as No. (\%) unless otherwise indicated, percentages are calculated on the basis of each group.

${ }^{\mathrm{b}}$ Percentage of initial drainage volume per CT volume $<50 \%$

${ }^{c}$ Percentage of initial drainage volume per CT volume $\geq 50 \%$

${ }^{\mathrm{d}}$ Data are means \pm standard deviations.

hancement on contrast-enhanced CT and magnetic resonance imaging as secondary findings due to inflammatory hyperemia. Kim et al. (16) reported that the parenchyma changes to immature pus and debris through the rapid invasion and destruction of the liver parenchyma in cases of KHA, and that the septa between the clusters of abscesses can be destroyed via inflammation as the abscess grows in size. Meanwhile, in non-KHA cases, the abscesses are not combined together and the structure is maintained as the septa are rarely destroyed. Hence, we believe that KHA cases exhibit hair ball-like abscess content with less frequent rim enhancement. Although our research results showed that the KHA group commonly exhibits a multiloc- ulated configuration, indicating septal breakage as per the research of Kim et al., the findings were not significant.

The mean attenuation of a hepatic abscess is the quantitative value that represents the liquefaction, and has been used to predict PCD failure by Liao et al. (14), who were the first to estimate this parameter. Liao et al. reported that the minimal attenuation values of abscesses, range of attenuation values, lack of a cystic component, and presence of gas were findings associated with PCD failure. However, the authors had measured the attenuation of the whole area, including gas as the measurement of attenuation values of the area while excluding gas was considered to be difficult. Hence, to obtain the mean attenuation values, 
Table 5. Clinical Outcomes Between Groups ${ }^{\mathrm{a}}$

\begin{tabular}{|c|c|c|c|c|}
\hline & Duration of Drainage (Days) & $\begin{array}{l}\text { Number of Days for Which the } \\
\text { Fever had Subsided (Days) }\end{array}$ & $\begin{array}{l}\text { Number of Days for Which } \\
\text { Leukocytosis had Subsided (Days) }\end{array}$ & Length of Hospitalization (Days) \\
\hline Group $A^{b}$ & $7.1 \pm 5.7$ & $5.8 \pm 3.5$ & $5.1 \pm 6.2$ & $25.4 \pm 13.6$ \\
\hline Group B ${ }^{c}$ & $8.7 \pm 7.2$ & $5.3 \pm 5.4$ & $6.1 \pm 6.6$ & $24.3 \pm 11.1$ \\
\hline PValue & 0.248 & 0.640 & 0.454 & 0.706 \\
\hline Group C ${ }^{b}$ & $7.3 \pm 6.4$ & $6.1 \pm 3.7$ & $5.5 \pm 6.7$ & $26.2 \pm 10.8$ \\
\hline Group $D^{c}$ & $8.1 \pm 4.8$ & $5.5 \pm 3.7$ & $4.9 \pm 4.2$ & $20.7 \pm 7.7$ \\
\hline P Value & 0.619 & 0.511 & 0.663 & 0.026 \\
\hline Group $E^{b}$ & $6.5 \pm 3.0$ & $4.9 \pm 2.7$ & $3.9 \pm 4.5$ & $23.0 \pm 19.9$ \\
\hline Group $F^{c}$ & $10.0 \pm 10.5$ & $5.1 \pm 7.9$ & $8.4 \pm 9.5$ & $31.2 \pm 13.4$ \\
\hline PValue & 0.229 & 0.943 & 0.111 & 0.251 \\
\hline
\end{tabular}

${ }^{\mathrm{a}}$ Values are expressed as mean $\pm \mathrm{SD}$.

${ }^{b}$ Percentage of initial drainage volume per CT volume $<50 \%$.

${ }^{\mathrm{c}}$ Percentage of initial drainage volume per CT volume $\geq 50 \%$.

we measured the area while excluding gas, and separately indicated whether gas was included. The abscess with a greater cystic portion is generally expected to have lower value for mean attenuation and larger drainage percent. Liao et al. also found that a larger cystic component is associated with successful PCD, and presence of a cystic component ensures high-quality drainage. In the present study, we could quantitatively identify a negative correlation between the percentage of initial drainage volume per CT volume and mean attenuation. Moreover, group B (percentage of initial drainage volume per CT volume $\geq 50 \%$ ) showed a significantly lower value for mean attenuation of the hepatic abscesses, as compared to that in group A (percentage of initial drainage volume per CT volume < 50\%).

In the histopathological analysis of pyogenic abscesses, the abscess cavity may show multiple locules that are usually filled with thick and purulent material and lined by pale fibrous tissue. The fibrous cuff around the abscess is often $\geq 1 \mathrm{~cm}$ thick and gradually merges into the liver parenchyma (17). Lee et al. reported that the KHA group tends to have thin walls whereas the non-KHA group tends to have thick walls, which may be due to the differences in the rate of invasion and destruction as a result of the presence of different microorganisms in the hepatic parenchyma $(4,13,16)$. In the present study, group B (percentage of initial drainage volume per CT volume $\geq 50 \%$ ) showed a significantly thicker abscess wall, and the KHA group with good drainage (group D) exhibited the same finding. This cannot be explained only by the destruction rate due to the presence of different microorganisms. Based on our experience with the clinical progression of pyogenic abscesses, even if the abscess wall is clearly visible in the active stage, it often disappears during regression. This finding may be associated with the variation in maturation and liquefaction according to the abscess stage.

Liao et al. (14) found that the shortest length to the liver capsule of $<0.25 \mathrm{~cm}$, abscess size $>7.3 \mathrm{~cm}$, a wide range of attenuation values, lack of a cystic component, lower minimal attenuation value, and the presence of gas were predisposing factors for PCD failure in pyogenic abscess patients, whereas other studies indicated that advanced age, respiratory failure, coagulopathy, and it has been reported that sonographic appearance of the abscess (large size and multiloculation) reduces the PCD success rate (10, 12). However, no studies have quantitatively examined the abscess volumes thus far. Moreover, the extent of drainage required for hepatic abscesses is unclear. The present study is the first to determine the hepatic abscess volumes quantitatively by using CT scan images and to evaluate the correlation between $\mathrm{CT}$ findings and clinical outcomes after categorizing patients based on the percentage of initial drainage volume per CT volume.

This study had certain limitations. First, the possibility of selection bias that could have resulted from the retrospective design of our study should be considered. Second, since there are no studies on CT volume and the percentage of initial drainage volume per CT volume, it is difficult to find a reference to justify the arbitrary division between good drainage and poor drainage groups by using the percentage value of initial drainage volume per CT volume of 50\%. Liao et al. categorized the patients with hepatic abscess into two groups, failure group and non-failure group, according to PCD treatment outcome (14). There were few patients in whom PCD therapy had failed in our study (three were dead and three underwent surgical in- 
tervention out of 87 patients). So, it was not an appropriate method of categorization. Therefore, further studies are required on this subject. Third, the statistical analysis was limited as there were fewer subjects in the non-KHA group. Fourth, automatic segmentation is generally based on difference of densities between the mass and normal hepatic parenchyma. Because the hepatic abscess is consisted of not only the cystic portion but also the solid portion, it was difficult to define the outer margin of the abscess automatically. That is the reason why we used freehand drawing by consensus of visual inspection between the radiologists. Using dedicated software for CT volumetric measurements might provide more precise results. Finally, we did not measure the total volume of pus drained after the initial drainage, and did not evaluate secondary procedures such as tube change or additional tube insertion, which could have reduced the accuracy of the clinical outcome evaluation in the patients.

In this study, the implication for patient care is that it could be helpful for making decisions about treatment timing and management strategies including follow-up interval, intensive medical therapy, or surgical intervention of hepatic abscess based on our CT imaging findings. And the result of our study also supports the previous finding that percutaneous drainage is effective in the treatment of hepatic abscesses, regardless of whether they are multiloculated. In conclusion, the less frequent occurrence of rim enhancement on CT may serve as an indicator of KHA and the presence of a thick wall on CT may serve as a predictor of a greater percentage of initial drainage amount per CT volume during PCD or PNA in patients with hepatic abscesses and a tendency of shorter length of hospital stay. Hence, CT findings may be useful for predicting the outcome of PCD or PNA in patients with hepatic abscess.

\section{Acknowledgments}

None declared.

\section{Footnotes}

Authors' Contributions: All authors contributed extensively to the study.

Financial Disclosure: There was no financial disclosure for the research.

Funding/Support: There was no funding support for the research.

\section{References}

1. Lederman ER, Crum NF. Pyogenic liver abscess with a focus on Klebsiella pneumoniae as a primary pathogen: an emerging disease with unique clinical characteristics. Am J Gastroenterol. 2005;100(2):322-31. doi: 10.1111/j.1572-0241.2005.40310.x. [PubMed: 15667489].

2. Yang CC, Yen $\mathrm{CH}$, Ho MW, Wang JH. Comparison of pyogenic liver abscess caused by non-Klebsiella pneumoniae and Klebsiella pneumoniae. J Microbiol Immunol Infect. 2004;37(3):176-84. [PubMed: 15221038].

3. Alsaif HS, Venkatesh SK, Chan DS, Archuleta S. CT appearance of pyogenic liver abscesses caused by Klebsiella pneumoniae. Radiology. 2011;260(1):129-38. doi: 10.1148/radiol.11101876. [PubMed: 21460028].

4. Lee NK, Kim S, Lee JW, Jeong YJ, Lee SH, Heo J, et al. CT differentiation of pyogenic liver abscesses caused by Klebsiella pneumoniae vs non-Klebsiella pneumoniae. Br J Radiol. 2011;84(1002):518-25. doi: 10.1259/bjr/23004588. [PubMed: 21081584].

5. Agarwal DK, Baijal SS, Roy S, Mittal BR, Gupta R, Choudhuri G. Percutaneous catheter drainage of amebic liver abscesses with and without intrahepatic biliary communication: a comparative study. Eur J Radiol. 1995;20(1):61-4. [PubMed: 7556257].

6. Attar B, Levendoglu H, Cuasay NS. CT-guided percutaneous aspiration and catheter drainage of pyogenic liver abscesses. Am J Gastroenterol. 1986;81(7):550-5. [PubMed: 3717117].

7. Gerzof SG, Johnson WC, Robbins AH, Nabseth DC. Intrahepatic pyogenic abscesses: treatment by percutaneous drainage. Am J Surg. 1985;149(4):487-94. [PubMed: 3885778].

8. Saraswat VA, Agarwal DK, Baijal SS, Roy S, Choudhuri G, Dhiman RK, et al. Percutaneous catheter drainage of amoebic liver abscess. Clin Radiol. 1992;45(3):187-9. [PubMed: 1555372].

9. Singh JP, Kashyap A. A comparative evaluation of percutaneous catheter drainage for resistant amebic liver abscesses. Am J Surg. 1989;158(1):58-62. [PubMed: 2662790].

10. Huang CJ, Pitt HA, Lipsett PA, Osterman FAJ, Lillemoe KD, Cameron JL, et al. Pyogenic hepatic abscess. Changing trends over 42 years. Ann Surg. 1996;223(5):600-7. [PubMed: 8651751] discussion 607-9.

11. Malangoni MA, Shumate CR, Thomas HA, Richardson JD. Factors influencing the treatment of intra-abdominal abscesses. Am J Surg. 1990;159(1):167-71. [PubMed: 2294795].

12. Tan YM, Chung AY, Chow PK, Cheow PC, Wong WK, Ooi LL, et al. An appraisal of surgical and percutaneous drainage for pyogenic liver abscesses larger than 5 cm. Ann Surg. 2005;241(3):485-90. [PubMed: 15729072].

13. Hui JY, Yang MK, Cho DH, Li A, Loke TK, Chan JC, et al. Pyogenic liver abscesses caused by Klebsiella pneumoniae: US appearance and aspiration findings. Radiology. 2007;242(3):769-76. doi: 10.1148/radiol.2423051344. [PubMed: 17325065].

14. Liao WI, Tsai SH, Yu CY, Huang GS, Lin YY, Hsu CW, et al. Pyogenic liver abscess treated by percutaneous catheter drainage: MDCT measurement for treatment outcome. Eur J Radiol. 2012;81(4):609-15. doi: 10.1016/j.ejrad.2011.01.036. [PubMed: 21330079].

15. Landis JR, Koch GG. The measurement of observer agreement for categorical data. Biometrics. 1977;33(1):159-74. [PubMed: 843571].

16. Kim SB, Je BK, Lee KY, Lee SH, Chung HH, Cha SH. Computed tomographic differences of pyogenic liver abscesses caused by Klebsiella pneumoniae and non-Klebsiella pneumoniae. J Comput Assist Tomogr. 2007;31(1):59-65. doi: 10.1097/01.rct.0000224629.48068.69. [PubMed: 17259834].

17. Mortele KJ, Segatto E, Ros PR. The infected liver: radiologicpathologic correlation. Radiographics. 2004;24(4):937-55. doi: 10.1148/rg.244035719. [PubMed: 15256619]. 